\title{
A Low-Complexity Multiuser Detector for Asynchronous CDMA QPSK Systems
}

\author{
E. Del Re, R. Fantacci, S. Morosi, G. Vivaldi. \\ Dipartimento di Ingegneria Elettronica \\ Università di Firenze, Via di S.Marta 3, I-50139 Firenze, Italia \\ e-mail address:morosi@tmed.die.unifi.it
}

\begin{abstract}
This paper deals with a multiuser detector based on a new decorrelating approach for asynchronous up-link DS/CDMA communications in which the QPSK modulation scheme is used in transmission. It is shown here that the receiver proposed is near-far resistant and exhibits a low complexity which allows real-time operation. Performance is expressed in terms of bit error rate (BER) which has been derived by simulations under the assumption of a slowly frequencyselective Rayleigh fading channel.
\end{abstract}

\section{INTRODUCTION}

The usefulness of CDMA systems in mobile radio communications is now generally acknowledged. The Rake receiver is a widely used detection scheme in CDMA systems. However, it has been demonstrated that the conventional Rake receiver performance is impaired by the so-called near-far effect. Some researchers [1]-[3] have concentrated their efforts on designing multiuser detectors capable of reducing these negative phenomena. The resistance of these receivers to the near-far problem implies promising outlook for these systems in the field of personal communications. The main drawback here is the implementation complexity which has so far prevented their complete development. The implementation complexity of a multiuser detection scheme may be reduced by the sliding window decorrelating algorithm (SLWA) proposed by Wijayasuria et al.[2]. This is a practical and flexible approach suitable for applications where the number of users is high and changing with time. A modified version of the Wijayasuria et al.'s approach was recently proposed by Yoon et al. [3]. In this case the cross-correlation from the past and future symbols in the asynchronous channels is eliminated (see Fig. 1) by using the frame format and periodical insertion of a zero-energy bit in transmission. This correspondence deals with an extention of the Yoon et al.'s method to the case of a QPSK modulation scheme and slowly frequency selective Rayleigh communication channel. In particular, the QPSK scheme has been considered in order to allow a better bandwidth occupation with respect to the PSK scheme. Performance comparisons with the classical Rake receiver in terms of BER are shown in order to highligth the advantages of our detection approach.

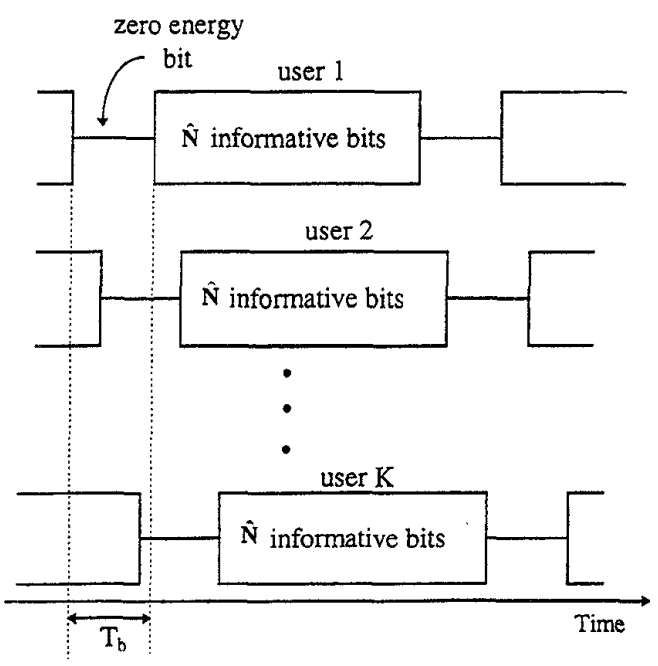

Fig. 1 : Transmitted data frame structure.

\section{SYSTEM MODEL}

A communication system with $\mathrm{K}$ simultaneous users is considered herein. Each user is assumed to transmit a packet of $\mathrm{N}$ bits. Let $\mathrm{n}(\mathrm{t})$ be the AWGN introduced by the communication channel under the assumption of a QPSK modulation scheme and of the use of the same spreading sequence for the phase and quadrature components (as is shown in Fig. 2), and thus we have [1]:

$$
\begin{gathered}
y_{l}(l)+j y_{Q}(l)=R(1) C(l+1)[i(l+1)+j q(l+1)]+R(0) C(l)[i(l)+ \\
j q(l)]+R(1) C(l-1)[i(l-1)+j q(l-1)]+n_{l}(l)+j \mathbf{n}_{\mathrm{Q}}(l)
\end{gathered}
$$

where $i(l)+j q(l)$ is the complex vector composed of the transmitted data. For example $i(l)=\left[i_{1}(l), i_{2}(l), \ldots, i_{\mathrm{KL}}(l)\right]$, where $i_{k}(l) \in\{ \pm 1\}$. 


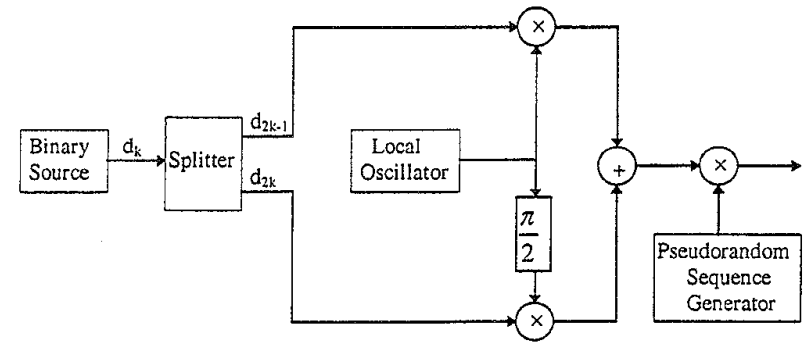

Fig. 2: Transmission system block diagram.

Each vector is comprised of $K L$ elements, where $L$ is the number of replicas of each user signal which can be discriminated at the receiving end. Each replica is considered as an individual user, so that it is still possible to exploit multiuser detection and to take advantage of the Rake diversity technique. Then, $\boldsymbol{y}_{l}(l)+j y_{\ell}(l)$ is the complex vector composed of the matched filter outputs, $C(l)$ are the diagonal matrices whose components are the complex channel fading gains of average energy $E_{i}$ :

$$
c_{k}(l)=\sqrt{w_{k}(l)} e^{j \theta_{k}(l)}
$$

$\boldsymbol{R}(l)$ are the $\mathrm{KL} \times \mathrm{KL}$ normalized signal cross-correlation matrices defined in [1] and $n_{t}(l)+j \mathbf{n}_{Q}(l)$ is due to the additive white Gaussian channel noise (AWGN) with zero mean and one-sided power spectral density equal to $\mathrm{N}_{0}$. Since $C(l)$ are complex matrices, it is possible to assume:

$$
C(l)=C_{l}(l)+\mathrm{j} C_{Q}(l)
$$

Thus, the complex vectorial equation (1) may be decomposed in the two real vectorial equations:

$$
\begin{gathered}
y_{t}(l)=R(-1)\left[C_{l}(l+1) i(l+1)-C_{\ell}(l+1) q(l+1)\right]+R(0)\left[C_{l}(l) i(l)-\right. \\
\left.C_{\ell}(l) q(l)\right]+R(1)\left[C_{l}(l-1) \dot{i}(l-1)-C_{\varrho}(l-1) q(l-1)\right]+n_{t}(l)
\end{gathered}
$$

$y_{Q^{\prime}}(l)=R(-1)\left[C_{l}(l+1) q(l+1)+C_{Q}(l+1) i(l+1)\right]+R(0)\left[C_{l}(l) q(l)+\right.$

$$
\left.C_{Q}(l) i(l)\right]+R(1)\left[C_{I}(l-1) i(l-1)+C_{Q}(l-1) q(l-1)\right]+\mathbf{n}_{Q}(l)
$$

where vectors $i(l)$ and $q(l)$ are unknown and have to be derived. To this end, we define:

$$
\left\{\begin{array}{l}
I(l)=C_{I}(l) i(l)-C_{\ell}(l) q(l) \quad \forall l=-\mathrm{M}, \ldots, \mathrm{M} \\
Q(l)=C_{l}(l) q(l)+C_{\ell}(l) i(l)
\end{array}\right.
$$

By trivial substitution in (2) and (3), we have:

$$
\left\{\begin{aligned}
\mathbf{y}_{\mathrm{t}}(l)=\mathbf{R}(-1) \mathbf{I}(l+1)+\mathbf{R}(0) \mathbf{I}(l) & +\mathbf{R}(1) \mathrm{I}(l-1)+\mathbf{n}_{\mathrm{I}}(l) \\
\mathbf{y}_{\mathrm{Q}}(l)=\mathbf{R}(-1) \mathbf{Q}(l+1)+\mathbf{R}(0) \mathbf{Q}(l) & +\mathbf{R}(1) \mathbf{Q}(l-1)+\mathbf{n}_{\mathrm{Q}}(l) \\
& \forall l=-\mathrm{M}, \ldots, \mathrm{M}
\end{aligned}\right.
$$

Hereinafter, we focus on the first equation in (5). We will extend later the results obtained to the second equation. We considered a sequence formed by $\hat{N}$ bits (window) for each user. From (5), by means of some algebraic manipulations, we have:

$$
\begin{aligned}
{\left.\left[\begin{array}{c}
\mathbf{y}_{\mathrm{I}}(M+1) \\
\mathbf{y}_{\mathrm{I}}(M+2) \\
\mathbf{y}_{1}(M+3) \\
\vdots \\
\mathbf{y}_{\mathrm{I}}(M+\hat{N})
\end{array}\right]=\left[\begin{array}{ccccc}
\mathbf{R}(0) & \mathbf{R}(-1) & 0 & \cdots & 0 \\
\mathbf{R}(1) & \mathbf{R}(0) & \mathbf{R}(-1) & \cdots & 0 \\
0 & \mathbf{R}(1) & \mathbf{R}(0) & \cdots & 0 \\
\vdots & \vdots & \vdots & \vdots & \vdots \\
0 & \cdots & \cdots & \mathbf{R}(1) & \mathbf{R}(0)
\end{array}\right] \begin{array}{c}
\mathbf{I}(M+1) \\
\mathbf{I}(M+2) \\
\mathbf{I}(M+3) \\
\vdots \\
\mathbf{I}(M+\hat{N})
\end{array}\right]+ } \\
+\left[\begin{array}{c}
\mathbf{R}(1) \mathbf{I}(M) \\
0 \\
\vdots \\
0 \\
\mathbf{R}(-1) \mathbf{I}(M+\hat{N}+1)
\end{array}\right]+\left[\begin{array}{c}
\mathbf{n}_{\mathrm{I}}(M+1) \\
\mathbf{n}_{\mathrm{I}}(M+2) \\
\mathbf{n}_{\mathbf{I}}(M+3) \\
\vdots \\
\mathbf{n}_{1}(M+\hat{N})
\end{array}\right]
\end{aligned}
$$

where $M$ is an offset from the start of the transmission and $\hat{N}<\mathrm{N}$ is the data temporal window length within the complete received sequence; the linear system (6) is composed of $\hat{N}$ equations with $\hat{N}+2$ unknown values; in particular, $y_{t}(\mathrm{M}+1)$ is dependent on $I(\mathrm{M})$ and, likewise, $y_{t}(\mathrm{M}+\hat{N})$ on $I(\mathrm{M}+\hat{N}+1)$. Thus, we need to know these values to solve the linear system ( 6 ). One possible solution to this problem is to assume $\mathrm{M}=0$ and $\hat{N}=\mathrm{N}$ (in this way $I(\mathrm{M})$ and $I(\mathrm{M}+\hat{N}+1)$ are both equal to zero), but this is equivalent to consider the entire received sequence. This particular case is considered in [1] where $R$ is a NKL XNKL matrix. This makes the inversion of $R$ not feasible for practical values of the sequence length $N$. Even the interesting solution proposed by Lupas and Verdú [1] based on the implementation of an LTI filter becomes too complex for large $\mathrm{KL}$ values and cannot be performed in real time by the receiver. In order to avoid this drawback, we have adopted here a suitable approach. Our strategy is to assume terms $I(\mathrm{M})$ and $I(\mathrm{M}+\hat{N}+1)$ in (6) both equal to zero. This condition is fullfilled by means of the periodical insertion of a zero-energy bit in the informative bit sequence. In this way, the interference due to the crosscorrelation of the actual symbols with the past and future symbols in the asynchronous channels can be eliminated (see Fig. 1). Then, parameter $\hat{N}$ has been chosen in order to obtain a feasible solution of the linear system (6).

From Fig. 1 it can be seen that the transmission is arranged so that the relative differences in arrival times of the zeroenergy bits at the base-station are smaller than the single-bit duration $T_{b}$, i.e.,

$$
\left|\tau_{k}-\tau_{l}\right| \leq \mathrm{T}_{\mathrm{b}}, \quad \forall k, l
$$

We note that the overhead needed to fullfill the synchronisation constraints is not too heavy to be unacceptable [3].

Now, the linear system (6) can be solved by exploiting the block tridiagonal structure of $R$ (as can be seen in [2]) thanks to the following algorithm [2]: 


\section{A. Sliding-Window Decorrelating Algorithm}

$$
\begin{gathered}
\mathbf{Z}_{1}=\mathbf{R}(0)^{-1} \mathbf{R}(-1) \\
\mathbf{Z}_{\mathrm{k}}=\left[\mathbf{R}(0)-\mathbf{R}(1) \mathbf{Z}_{\mathrm{k}-1}\right]^{-1} \mathbf{R}(-1) \quad \mathrm{k}=2,3, \ldots, \hat{N} \\
\mathbf{W}_{1}=\mathbf{R}(0)^{-1} \mathbf{y}_{\mathbf{1}}(\mathrm{M}+1) \\
\mathbf{W}_{\mathrm{k}}=\left[\mathbf{R}(0)-\mathbf{R}(1) \mathbf{Z}_{\mathrm{k} \cdot \mathrm{l}}\right]^{-1}\left[\mathbf{y}_{\mathbf{l}}(\mathrm{M}+\mathrm{k})-\mathbf{R}(1) \mathbf{W}_{\mathrm{k} \cdot \mathrm{l}}\right] \quad \mathrm{k}=2,3, \ldots, \hat{N} \\
\mathbf{I}(\mathrm{M}+\hat{N})=\mathbf{W}_{\hat{\mathbf{N}}} \\
\mathbf{I}(\mathrm{M}+\mathrm{k})=\mathbf{W}_{\mathrm{k}}-\mathbf{Z}_{\mathrm{k}} \mathbf{I}(\mathrm{M}+\mathrm{k}+1) \quad \mathrm{k}=\hat{N}-1, \hat{N}-2, \ldots, 1
\end{gathered}
$$

Thus, all the values of vectors $\mathbf{I}(l)$ are determined. Then, $\mathbf{Q}(l)$ is obtained by solving a similar linear system. This means that two decorrelation blocks must be used.

After all these iterations, the mutual interference among users has been eliminated, but the decorrelation block outputs are dependent on the random phase offset introduced by the multipath fading channel. Hence, a phase compensation block has been introduced. In particular, the $k$-th element of (4) is equal to:

$$
\left\{\begin{array}{c}
\mathrm{I}_{\mathrm{k}}(l)=\sqrt{\mathrm{w}_{\mathrm{k}}(l) \mathrm{i}_{\mathrm{k}}}(l) \cos \vartheta_{\mathrm{k}}(l)-\sqrt{\mathrm{w}_{\mathrm{k}}(l)} \mathrm{q}_{\mathrm{k}}(l) \sin \vartheta_{\mathrm{k}}(l) \\
\mathrm{Q}_{\mathrm{k}}(l)=\sqrt{\mathrm{w}_{\mathrm{k}}(l)} \mathrm{q}_{\mathrm{k}}(l) \cos \vartheta_{\mathrm{k}}(l) \\
+\sqrt{\mathrm{w}_{\mathrm{k}}(l)} \mathrm{f}_{\mathrm{k}}(l) \sin \vartheta_{\mathrm{k}}(l) \\
\forall l=-\mathrm{M}, \ldots, \mathrm{M}
\end{array}\right.
$$

where $w_{k}(l)$ is the received power from the $l$-th bit of the $\mathrm{k}$ th user and $\vartheta_{k}(l)$ is the random phase introduced by the multipath fading channel. The perfect knowledge of $\vartheta_{k}(l)$ is assumed, while $w_{k}(l)$ has an unknown value. As can be easily seen, (7) is a simple linear system composed of two equations with two unknown values, $\sqrt{\mathrm{w}_{\mathrm{k}}(l)} \mathrm{i}_{\mathrm{k}}(l)$ and $\sqrt{\mathrm{w}_{k}(l)} \mathrm{q}_{\mathrm{k}}(l)$. Its solution is given by the following operations:

\section{B. Phase Compensation}

$$
\left\{\begin{aligned}
& \sqrt{\mathrm{w}_{\mathrm{k}}(l)} i_{\mathrm{k}}(l)=\mathrm{I}_{\mathrm{k}}(l) \cos \vartheta_{\mathrm{k}}(l)+\mathrm{Q}_{\mathrm{k}}(l) \sin \vartheta_{\mathrm{k}}(l) \\
& \sqrt{\mathrm{w}_{\mathrm{k}}(l)} \mathrm{q}_{\mathrm{k}}(l)=\mathrm{Q}_{\mathrm{k}}(l) \cos \vartheta_{\mathrm{k}}(l)-\mathrm{I}_{\mathrm{k}}(l) \sin \vartheta_{\mathrm{k}}(l) \\
& \forall l=-\mathrm{M}, \ldots, \mathrm{M}
\end{aligned}\right.
$$

In this way all the values of vectors $\mathbf{i}(l)$ are determined. Then, $\mathbf{q}(l)$ is obtained by solving a similar linear system.

It should be emphasised that the simplicity of this solution is due to our choice of using only one pseudo-noisy sequence for each QPSK user.

Finally, the values of the decision variables are obtained. These values are affected only by the AWGN and the attenuation given by the multipath fading, but they are completely free from the interference caused by the other users and the random phase deviation introduced by the multipath fading channel.

\section{SIMULATIONS RESULTS}

In this section the performance of the conventional Rake detector and that of the proposed receiver are compaired in different interference environments. Moreover, the dependence of the BER performance on the frame length is investigated. In performing our simulations the following conditions have been assumed:

- Symbol rate for the QPSK modulation equal to 31.496 ksymbols/sec;

- One spreading sequence assigned to each QPSK user (see Fig.2);

- Spreading obtained through Gold sequences with processing gain equal to 127 .

We consider 10 users and an up-link Rayleigh multipath fading channel with six resolvable paths and a Doppler spread equal to $100 \mathrm{~Hz}$. Figs. 3-5 show the BER performance of the proposed multiuser detector as a function of the ratio between the energy per bit at the receiving end $\left(E_{b}\right)$ and $N_{0}$, for different values of the power unbalance between the desired user signal and the interfering signals. The BER performance obtained for a classical Rake receiver with the same number of interfering users and a classical Rake receiver with a single user is also shown in the figures for comparison purposes. The good behaviour of the multiuser detector is apparent in Figs. 3-5. Of particular interest is the fact that the BER performance of the proposed multiuser detector is independent of the near-far effect.

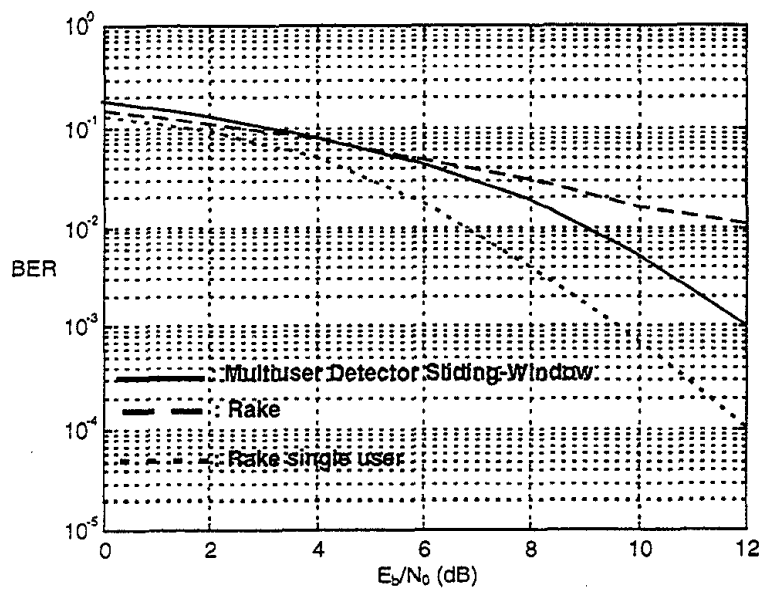

Fig. 3: Bit Error Rate comparisons (ideal power control). 


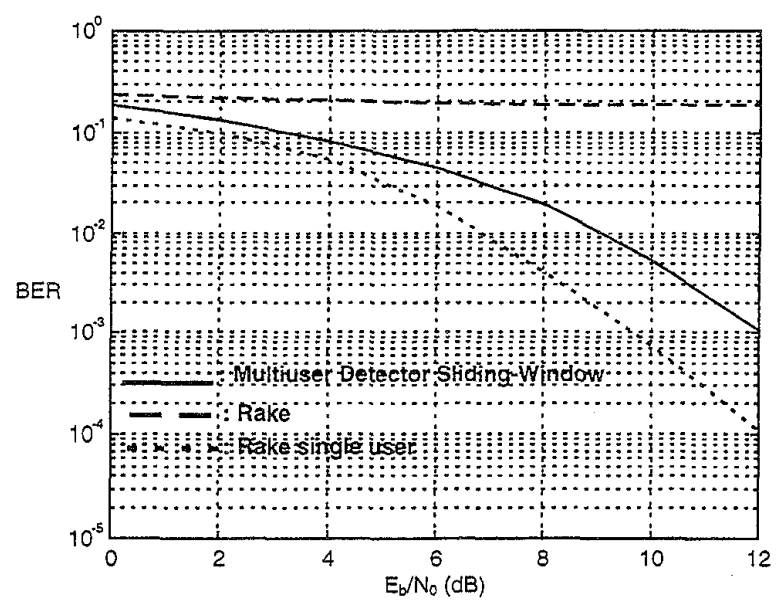

Fig.4: Bit Error Rate comparisons (power unbalance between desired user signal and interfering signals equal to $10 \mathrm{~dB}$ ).

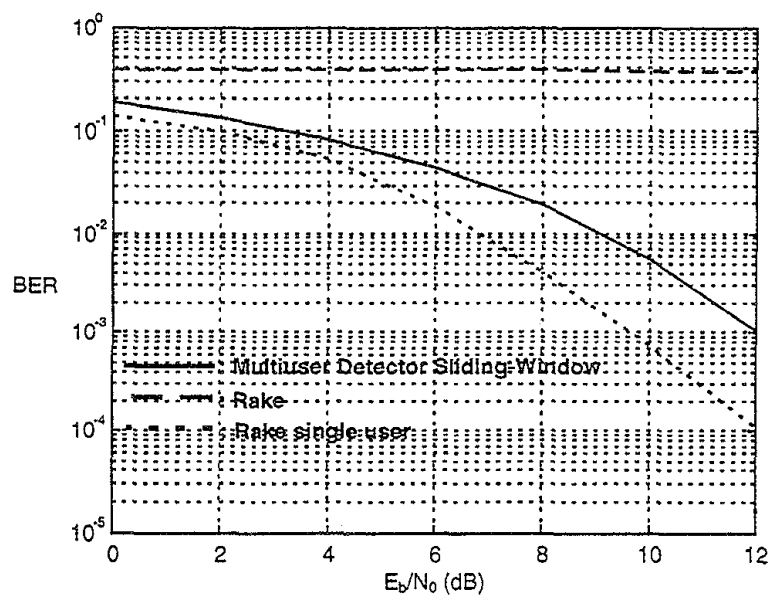

Fig.5: Bit Error Rate comparisons (power unbalance between desired user signal and interfering signals equal to $20 \mathrm{~dB}$ ).

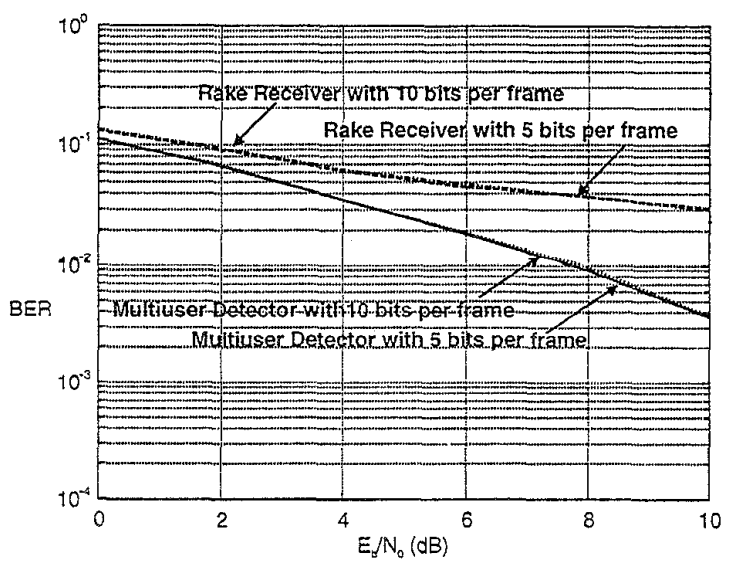

Fig. 6: Bit Error Rate comparisons (power unbalance between the desired user signal and the interfering signals equal to $10 \mathrm{~dB}$, with different frame lengths).
The results shown in Figs. 3-5 have been derived by inserting a zero-energy symbol every four information bits $(\hat{N}=4)$. This leads to an efficiency equal to 80 per cent. This value is clearly too low for practical applications. The dependence of the BER performance of the proposed receiver on the frame length is investigated in Fig. 6 for the case of four interfering users and three resolvable paths. The power unbalance between the desired user signal and the interfering signals has been set to $10 \mathrm{~dB}$. This figure proves that the BER performance is not dependent on the frame length; hence, high efficiencies are guaranteed.

\section{CONCLUSIONS}

In this paper In this correspondence a multiuser receiver for DS/CDMA up-link communications has been presented. The near-far resistance of our receiver and its good behaviour with respect to the classical Rake receiver have been verified by means of computer simulations in the case of a slowly frequency-selective Rayleigh fading channel.a multiuser receiver using a multi-element

\section{REFERENCES}

[1] R. Lupas, S. Verdù, "Near-Far Resistance of Multiuser Detectors in Asynchronous Channels", IEEE Trans. On Comm., Vol. COM-38, pp. 496-508, April 1990.

[2] S.S.H. Wijayasuria, G.H. Norton, J.P. McGeehan, "Sliding window decorrelating algorithm for DS-CDMA receivers", Electronics Letters, Vol. 28, No. 17, pp. 1596-1598, August 1992.

[3] R.E. Donovan, S.Y. Yoon, S.E. Hong, J. Ahn, H.S. Lee, "Pilot Symbol Aided Coherent Decorrelating Detector for Up-link CDMA Mobile Radio Communication", Electronics Letters, Vol. 30, No. 12, pp. 929-930, June 1994. 